University of Nebraska - Lincoln

DigitalCommons@University of Nebraska - Lincoln

USDA National Wildlife Research Center - Staff Publications
U.S. Department of Agriculture: Animal and Plant Health Inspection Service

2010

\title{
Seeking a Second Opinion: Uncertainty in Disease Ecology
}

\author{
Brett T. McClintock \\ USGS Patuxent Wildlife Research Center \\ James D. Nichols \\ USGS Patuxent Wildlife Research Center \\ Larissa L. Bailey \\ Colorado State University, Fort Collins, Department of Fish, Wildlife, and Conservation Biology \\ Darryl I. MacKenzie \\ Proteus Wildlife Research Consultants, PO Box 5193, Dunedin, New Zealand \\ William. L. Kendall \\ USGS Patuxent Wildlife Research Center \\ See next page for additional authors
}

Follow this and additional works at: https://digitalcommons.unl.edu/icwdm_usdanwrc

Part of the Environmental Sciences Commons

McClintock, Brett T.; Nichols, James D.; Bailey, Larissa L.; MacKenzie, Darryl I.; Kendall, William. L.; and Franklin, Alan B., "Seeking a Second Opinion: Uncertainty in Disease Ecology" (2010). USDA National Wildlife Research Center - Staff Publications. 944.

https://digitalcommons.unl.edu/icwdm_usdanwrc/944

This Article is brought to you for free and open access by the U.S. Department of Agriculture: Animal and Plant Health Inspection Service at DigitalCommons@University of Nebraska - Lincoln. It has been accepted for inclusion in USDA National Wildlife Research Center - Staff Publications by an authorized administrator of DigitalCommons@University of Nebraska - Lincoln. 


\section{Authors}

Brett T. McClintock, James D. Nichols, Larissa L. Bailey, Darryl I. MacKenzie, William. L. Kendall, and Alan B. Franklin 


\section{IDEA AND}

\section{PERSPECTIVE Seeking a second opinion: uncertainty in disease ecology}

Brett T. McClintock, ${ }^{1 *}$ James D. Nichols, ${ }^{1}$ Larissa L. Bailey, ${ }^{2}$ Darryl I. MacKenzie, ${ }^{3}$ William. L. Kendall $^{1}$ and Alan B. Franklin ${ }^{4}$ ${ }^{1}$ USGS Patuxent Wildlife Research Center, 12100 Beech Forest Road, Laurel, MD 20708, USA

${ }^{2}$ Department of Fish, Wildlife, and Conservation Biology, Colorado State University, Fort Collins, CO 80523, USA

${ }^{3}$ Proteus Wildlife Research Consultants, PO Box 5193, Dunedin, New Zealand ${ }^{4}$ USDA Animal and Plant Health Inspection Service, Wildlife Services, National Wildlife Research Center, 4101 La Porte Avenue, Fort Collins, CO 80521, USA

*Correspondence: E-mail: brett.mcclintock@gmail.com

\begin{abstract}
Analytical methods accounting for imperfect detection are often used to facilitate reliable inference in population and community ecology. We contend that similar approaches are needed in disease ecology because these complicated systems are inherently difficult to observe without error. For example, wildlife disease studies often designate individuals, populations, or spatial units to states (e.g., susceptible, infected, post-infected), but the uncertainty associated with these state assignments remains largely ignored or unaccounted for. We demonstrate how recent developments incorporating observation error through repeated sampling extend quite naturally to hierarchical spatial models of disease effects, prevalence, and dynamics in natural systems. A highly pathogenic strain of avian influenza virus in migratory waterfowl and a pathogenic fungus recently implicated in the global loss of amphibian biodiversity are used as motivating examples. Both show that relatively simple modifications to study designs can greatly improve our understanding of complex spatio-temporal disease dynamics by rigorously accounting for uncertainty at each level of the hierarchy.
\end{abstract}

\section{Keywords}

Host and pathogen dynamics, imperfect detection, incidence, misclassification, observation error, occupancy, presence-absence, prevalence, spatial epidemiology, species occurrence.

Ecology Letters (2010) 13: 659-674

\section{INTRODUCTION}

Wildlife disease ecology has received considerable recent attention because of the emergence and re-emergence of a number of pathogens causing disease in both humans and livestock (Dobson \& Foufopoulos 2001; Ostfeld \& Holt 2004; Webster et al. 2006). For example, about 60\% of all infectious diseases and $72 \%$ of recent emerging infectious diseases resulted from pathogens of wildlife origin (Taylor et al. 2001; Jones et al. 2008). Gaining understanding of infectious disease in wild populations is therefore critical not only for conservation, but also for human and agricultural health; however, the study of these complicated systems is inherently difficult. To help gain understanding of these systems, a wealth of epidemiological models has been developed, including spatio-temporal models focusing on the spread of infections and disease (e.g., Hudson et al. 2002; Keeling \& Rohani 2007). Although theoretical models offer useful predictions about the ecological characteristics of pathogens in natural populations, available data are often inadequate to accurately parameterize theoretical and risk assessment models. Indeed, Barlow (1995) found that half of the nearly three dozen wildlife disease models reviewed did not provide evidence that their predictions could be supported by empirical data. In another review, Gulland (1995) summarized the situation: 'The paucity of understanding of wildlife disease epidemiology thus arises...from the traditional approach to investigating disease in wildlife and the difficulties involved in collecting such information'. Wobeser (2006) likened disease in wild populations to 'an iceberg in that only a tiny tip projects above the water to be visible', and there have been repeated calls to improve surveillance programs for detecting disease and understanding its role in population dynamics and local species extinction (Plowright 1988; Smith et al. 2006; Yasué et al. 2006; Norman 2008; Nusser et al. 2008).

Two areas of disease ecology where reliable parameter estimation is critical are wildlife surveillance programs and 
the modelling of disease dynamics. Surveillance and monitoring programs tend to focus on the status of pathogens and disease in populations, with infection prevalence the primary parameter of interest (Salman 2003; Wobeser 2006). Modelling of pathogen and disease dynamics typically focuses on: (1) the cycling of pathogens and disease (e.g., incidence and transmission rates) within a host system; and (2) the potential for these pathogens to affect population dynamics and transmit to humans or livestock (e.g., Clark \& Hall 2006). These disease-dynamic models generally operate at two different scales: dynamics within host populations, and dynamics across space and host populations. Regardless of scale, disease ecologists are often limited by an inability to directly observe the underlying process of scientific interest and must therefore rely on observable (but imperfect) data to make inferences. For example, studies within host populations often designate individuals to states (e.g., susceptible, infected, postinfected) and investigate the state-specific patterns and dynamics of the system (Packer et al. 2003). However, the uncertainty associated with state assignments due to non- detection or misclassification (see Table 1) remains largely ignored or unaccounted for in inference. Indeed, there appears to be a pattern emerging where the potential for observation error in wildlife disease ecology is acknowledged, but not addressed in sampling designs and statistical analyses (e.g., Schall et al. 2000; Joly \& Messier 2004; Atkinson et al. 2005; Olsen et al. 2006; Jourdain et al. 2007). Failing to directly address observation error has the potential to perpetuate misleading inferences about disease in natural systems (Faustino et al. 2004; Ward et al. 2006; Jennelle et al. 2007). Even the simplest estimators, such as those commonly used for prevalence, may be biased because imperfect detectability has not been accounted for (Conner et al. 2000).

Much attention in the capture-mark-recapture literature (e.g., Williams et al. 2002) has focused on dealing with observation error to facilitate more reliable inference in population ecology. This gradual shift over the past five decades has depended upon the eventual acknowledgment by population ecologists that indices based on counts do not provide a valid basis for making inferences about abundance

Table 1 Glossary of terms

Term Definition

Conditional probability The conditional probability of some event A given the occurrence of some other event $\mathrm{B}$ is written as $\mathrm{Pr}$ $(A \mid B)=\operatorname{Pr}(A$ and $B) / \operatorname{Pr}(B)$, where $\operatorname{Pr}(A$ and $B)$ is the joint probability of both $\mathrm{A}$ and $\mathrm{B}$ occurring.

Detection history A concise summary of presence/absence data under repeated sampling, where the detection or non-detection of a state at a sample unit is indicated by a ' 1 ' or ' 0 ', respectively. For example, a single-season detection history $\mathbf{h}_{j}=011$ indicates that three samples were collected from sample unit $j$ when the state of the sample unit was static. The state of interest (e.g., pathogen presence) was detected in the second and third samples, but not the first. The multiseason (e.g., year-to-year) detection history $\mathbf{h}_{j}=011000$ indicates three samples were collected from sample unit $j$ in seasons one and two, but the state of interest failed to be detected during sampling in season two. The state of the sample unit remains static within, but not necessarily between, seasons.

Incidence

The proportion of uninfected sample units that become newly infected by a pathogen or disease during a given period of time.

Independence If two events $\mathrm{A}$ and $\mathrm{B}$ are independent, then the probability of both events is the product of the probabilities of the two events: $\operatorname{Pr}(A$ and $B)=\operatorname{Pr}(A) \operatorname{Pr}(B)$.

Likelihood function A function indicating how likely a particular population is to produce an observed sample. The likelihood function for the population parameters $(\theta)$ given the observed data $(y)$ is written as $L(\theta \mid y)$.

Misclassification A sampling condition arising when a state is encountered, but assigned to the wrong state. A false positive test result is an example of a misclassification error.

Mutually exclusive If two events $\mathrm{A}$ and $\mathrm{B}$ are mutually exclusive, then the probability of either event is the sum of the probabilities of the two events: $\operatorname{Pr}(A$ or $B)=\operatorname{Pr}(A)+\operatorname{Pr}(B)$.

Non-detection Failing to detect a state, although present, because it was not encountered (e.g., during sampling and/or laboratory assay).

Prevalence The proportion of sample units that is infected by a pathogen or disease at a specific point in time.

Repeated sampling The collection of multiple observations (i.e., samples) about the state of a system during a period when the true state of the system is static. Repeated sampling can be used to inform the detection process incorporating different types of observation error.

State uncertainty Uncertainty arising when a quantity that describes the true attribute of a system cannot be perfectly observed due to non-detection or misclassification. In disease ecology, these attributes might include susceptible, infected, and post-infected states of individuals, populations, or spatial units. 
and related demographic parameters (Anderson 2001). Given the relatively new emphasis on wildlife disease ecology, it is therefore not surprising that the use of uncorrected data, such as time series of counts, is commonplace in studies aimed at providing inferences about disease effects, prevalence, and dynamics (e.g., Begon et al. 1998; Hudson et al. 1998; Mellor \& Rockwell 2006; Wallenstein et al. 2007; Smith et al. 2009). However, it has been recently recognized that multistate capture-markrecapture models incorporating imperfect detection and state uncertainty can be adapted to examine disease within host populations. Jennelle et al. (2007) described two systems, avian pox in a population of Serins (Serinus serinus) and Mycoplasma gallisepticum conjunctivitis in House Finches (Carpodacus mexicanus), where multistate models were used to account for imperfect detection. Failing to account for differential detection probabilities among infected and uninfected individuals resulted in respective positive and negative biases in estimates of disease prevalence and, in both cases, there can be completely spurious patterns in system dynamics. Also examining conjunctivitis in finches, Conn \& Cooch (2009) applied a multistate model incorporating partial observability to examine survival and transition probabilities between infected and susceptible states. Using this approach, they were able to explicitly acknowledge that disease state could not always be definitively diagnosed upon visual inspection, thereby avoiding potential misclassification and unnecessary data censoring. The difficult problem of false positive errors has only very recently begun to receive attention in the capture-mark-recapture literature (Lukacs \& Burnham 2005; Yoshizaki et al. 2009), but both Jennelle et al. (2007) and Conn \& Cooch (2009) recognized the importance of incorporating this type of observation error into their models of disease. False positive error rates are increasingly being quantified in clinical studies (e.g., Carey et al. 2006), and there is much promise for future research and model development in this area.

Taking these recent developments into consideration, we contend that a similar shift is called for at the broader scale of surveillance and disease dynamics modelling, across both space and host populations. In this paper, we demonstrate how recent developments in species occurrence models that incorporate imperfect detection and state uncertainty extend quite naturally to hierarchical spatial models of disease effects, prevalence, and dynamics. We first identify the potential sources of observation error and their implications for inference at each level of the observation process, from spatio-temporal allocation of field sampling efforts to laboratory practices. We then formulate a general hierarchical strategy using repeated sampling at each level of the hierarchy to address this uncertainty. To reinforce the key components of this alternative approach, illustrative examples focusing on real-world disease problems of immediate concern are utilized. These include a highly pathogenic strain of avian influenza virus in migratory waterfowl (Liu et al. 2005) and a pathogenic fungus recently implicated in the global loss of amphibian biodiversity (Wake \& Vredenburg 2008) as motivating examples of how this methodology can greatly improve our understanding of complex spatiotemporal disease dynamics in natural systems. In the face of substantial uncertainty, both examples emphasize that relatively simple modifications to the designs under which data are collected and analysed can facilitate broader and more reliable inferences in disease ecology.

\section{Sources of uncertainty in disease ecology}

Part of what makes reliable inference in disease ecology so difficult is the myriad of ways uncertainty can be introduced by observation error (Fig. 1). From the selection of spatial units, populations, or individuals for surveillance, to the collection, handling, and diagnosis of samples, uncertainty can enter at any or all levels of the process. To illustrate, consider uncertainty in the context of a hypothetical broadscale surveillance program for highly pathogenic Asian strain H5N1 avian influenza virus (HPAIV) among waterfowl (family Anatidae) in North America (Cattoli \& Capua 2007; Kendall 2009). Waterfowl and shorebirds serve as the natural reservoir of avian influenza viruses (AIV; Webster et al. 1992), and there is considerable concern about the current global spread of HPAIV because of its impact on poultry and its pandemic potential to humans. Although HPAIV has not been detected in the United States, concern about the potential introduction of this pathogen by migrating waterfowl resulted in the initiation of a nationwide wild bird surveillance program (U. S. Interagency Strategic Plan 2006). Although some North American waterfowl species can become fatally diseased with HPAIV, other species remain asymptomatic when infected, even though they are still infectious (Brown et al. 2006). Therefore, surveillance for HPAIV virus is not predicated on the detection of clinically suspect animals.

Given the virus were to arrive and were present in North America, there is uncertainty associated with the selection of geographic sample units because the selected units (or subunits therein) may not contain infected individuals (Fig. 1a, step 1). Given some individuals in a selected spatial unit are in fact infected, there is uncertainty associated with the sampling of animals because not all individuals may be infected (Fig. 1a, steps 2-3). Given a sampled individual is infected, there is uncertainty associated with detection of the virus in samples based on the assay technique (Fig. 1b). There are multiple levels of uncertainty in laboratory assays commonly used to detect HPAIV in samples, including the sensitivity and specificity of the PCR assays to initially detect AIV, subsequently identify the virus 
(a)

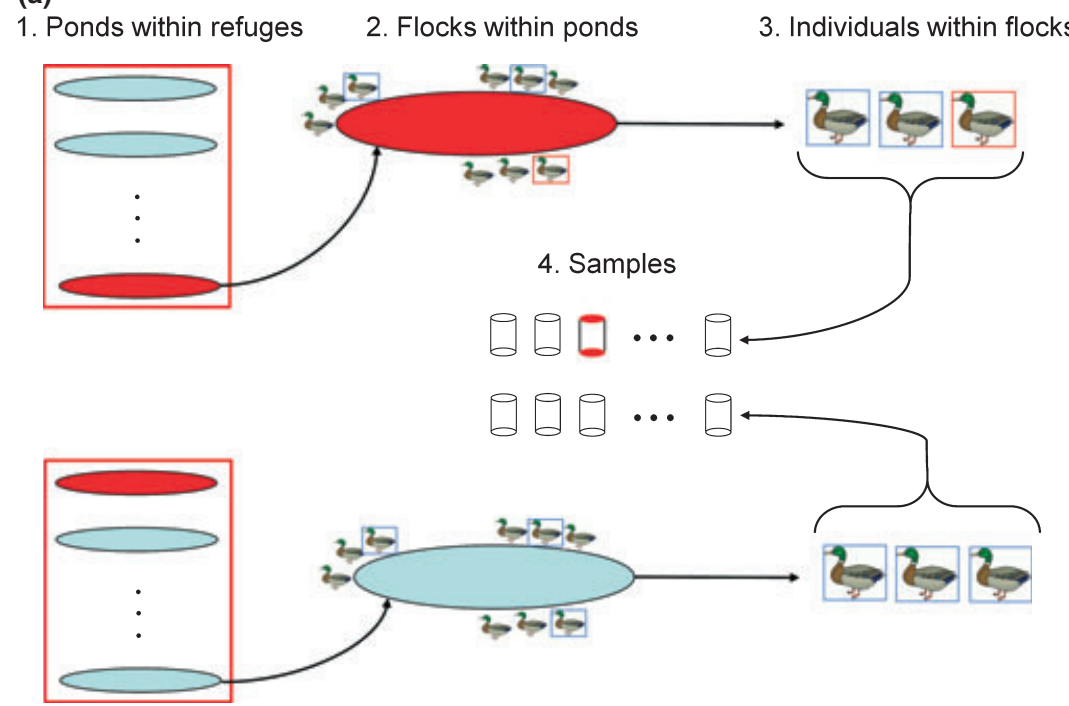

(b) 1. Samples

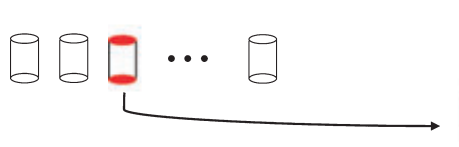

2. Extraction

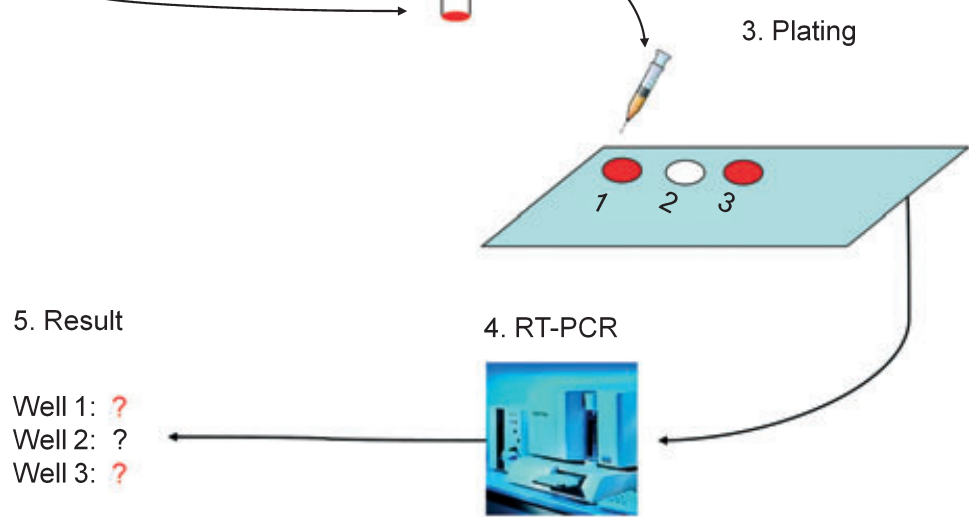

Figure 1 Conceptualization of the myriad ways in which uncertainty can emerge in wildlife disease ecology (e.g., avian influenza in waterfowl populations), from the spatiotemporal allocation of field sampling effort (a) to laboratory practices (b). Red indicates infected samples and sample units. Whether or not a sample is infected, false negative or false positive test results can conceivably occur. as an $\mathrm{H} 5$ subtype, and finally determine whether the H5 (if detected as such) is highly pathogenic. Finally, there is the additional uncertainty that any of the tests resulted in a false positive error, a form of misclassification that can arise due to cross-reaction with other subtypes, lack of specificity to AIV, or contamination. The incorporation of observation error is therefore important for estimating results of laboratory assays (i.e., by analysing duplicate subsamples; see Fig. 1b, steps 3-5) as well as evaluating methods used to detect pathogens of interest (e.g., Hui \& Walter 1980).

The levels of uncertainty in disease studies can thus be formulated in a hierarchical series of conditional probability statements (Table 1). Consider a hierarchy, where the levels of uncertainty fall under four general themes (Fig. 2):

Level 1: Pr (largest geographic area contains infected individuals).
Level 2: Pr (selected sample unit contains infected individuals | larger geographic area infected).

Level 3: Pr (selected individual infected | sample unit infected).

Level 4: (a) Pr (detect pathogen with assay | individual infected); (b) $\operatorname{Pr}$ (detect pathogen with assay | individual not infected).

Levels within these general themes can then be added or removed as dictated by the disease system, study design, and number of branches in the spatial hierarchy. Although Levels 1, 2, or 3 are typically of most ecological interest, uncertainty at all levels of the hierarchy needs to be adequately addressed to facilitate reliable inferences. In our HPAIV example, suppose interest lies in estimating prevalence at different levels of spatial coarseness (e.g., the spatial prevalence of HPAIV among ponds within wildlife refuges located in $10 \mathrm{~min}$ blocks). Additional levels could 
Figure 2 Hierarchical formulation of uncertainty in wildlife disease ecology under four general themes. Conditional on the disease state at the upper levels, many different sample paths can lead to a false negative or false positive result upon analysis at Level 4. Spatial subunits may be added or removed within Level 2 of the hierarchy.

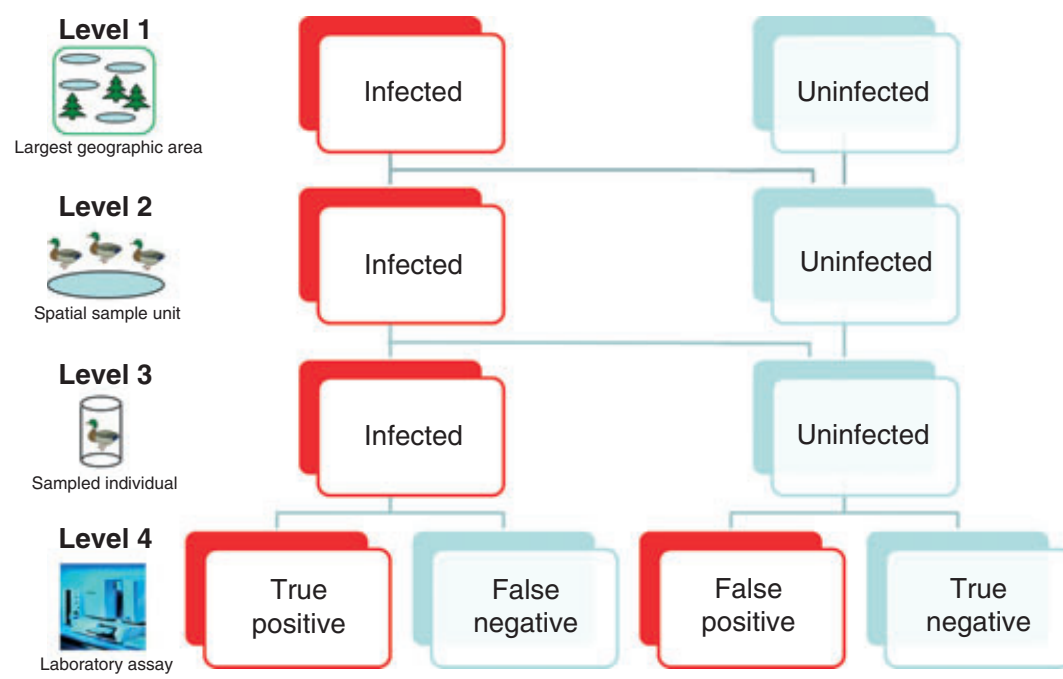

then be introduced for each spatial subunit (pond and refuge) within each 10 min block of the larger geographic region of interest (e.g., North America). Similarly, the probability of detecting HPAIV given the virus were present within an individual sample could consist of three levels (one for each of the three laboratory diagnostics), each with its own probability of a true or false positive detection.

Regardless of the hierarchical structure, targeted surveillance based on opportunistic (or 'convenience') sampling is particularly prone to misleading inferences (Conner et al. 2000; Nusser et al. 2008). We will therefore assume that a surveillance protocol has some basis in probability sampling and focus on state uncertainty arising within the hierarchy through non-detection and misclassification. If not accounted for, false negative (or false positive) errors will introduce negative (or positive) biases in estimates of prevalence and potentially spurious patterns in pathogen effects and disease dynamics (see Fig. 3). Laboratory diagnostics are typically optimized for sensitivity and specificity using known positive and negative samples, although these protocols do not guarantee accurate results (Cattoli \& Capua 2007; Chua et al. 2007; Hyatt et al. 2007; VanDalen et al. 2008). Careful selection and handling of specimens can also reduce the potential for these errors, but it is unlikely that sound field and laboratory protocols will eliminate all error. Despite this, it remains standard practice to ignore false negatives, false positives, and observability biases in the application of wildlife disease models to spatiotemporal data (e.g., Hartup et al. 2001; Hess et al. 2002; Norman 2008; Osnas et al. 2009).

\section{Hierarchical modelling of disease state uncertainty based on repeated sampling}

Our recommendation is that uncertainty at all levels of the hierarchy be directly incorporated into spatio-temporal disease models and informed by repeated sampling. This approach relies on adapting and extending models originally developed for examining patterns and dynamics of species occurrence, when species are detected imperfectly (Royle \& Link 2005; Nichols et al. 2008; MacKenzie et al. 2009). The fundamental basis for these models is that detection probabilities for species within spatial units can be estimated via repeated sampling over time or space, and we wish to demonstrate how this principle can be extended to a wide variety of problems in disease ecology.

Spatial prevalence and dynamics under imperfect detection

Consider an investigation of the prevalence of the pathogenic fungus [Batrachochytrium dendrobatidis $(B d)$ ] suspected in recent global amphibian declines (Pounds et al. 2006; Wake \& Vredenburg 2008) in a subset of wetlands within a national park (note that all models developed under this example could as easily be applied to the previous HPAIV example). Studies have suggested that $B d$ can exist in aquatic habitats for several weeks in the absence of host species (Johnson \& Speare 2003). Furthermore, several species can serve as $B d$ hosts, but only a subset of anuran species and populations seem particularly vulnerable to the disease (Muths et al. 2008). If a single water sample was collected from each wetland (Kirshtein et al. 2007), the data would consist of samples testing $B d$ positive (indicated by a ' 1 ') or $B d$ negative (indicated by a ' 0 '). However, suppose each wetland was sampled on three occasions (e.g., samples were collected on three different days or at three different locations within each wetland sensu Kendall \& White 2009) during a period where the $B d$ state of each wetland was static. Under perfect detection, the observed data would consist entirely of 111 and 000 detection histories (Table 1) for $B d$ positive and negative wetlands, respectively. However, false nega- 
(a)
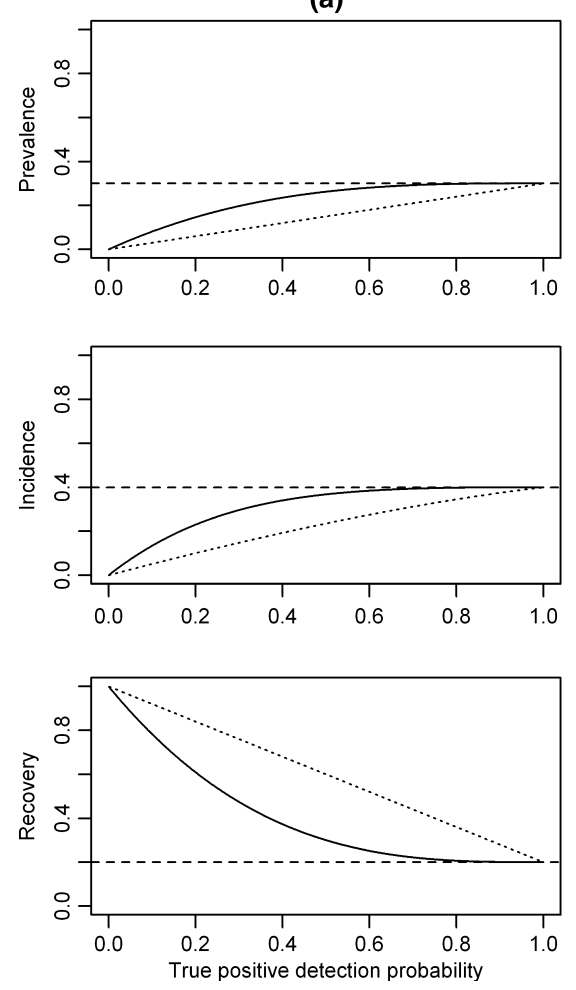

(b)
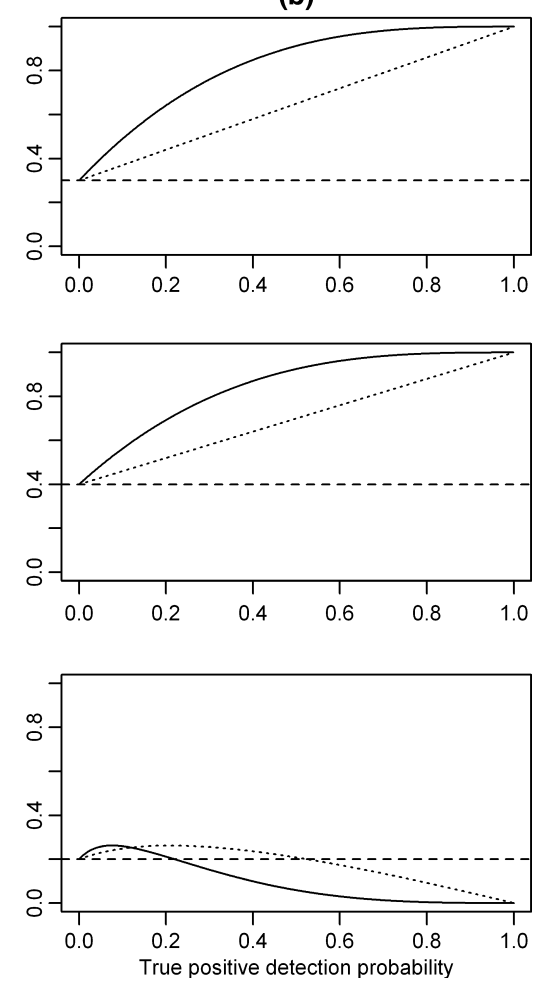

Figure 3 A simple numerical example of the types and magnitudes of bias that can result when imperfect detection is ignored in epidemiological studies. Plots are large-sample approximations for naïve estimators (i.e., assuming no false negative and no false positive detections) of the probability of a sample unit initially being infected (prevalence), the probability of a unit transitioning from uninfected to infected (incidence), and the probability of a unit transitioning from infected to uninfected (recovery). Expectations are plotted as a function of: (a) the probability of detecting infection, given presence of the pathogen within a unit, in a single sample when the probability of a false positive detection is zero; and (b) the probability of falsely detecting infection, given absence of the pathogen within a unit, in a single sample when the probability of a false negative detection is zero. Dashed lines represent the true values for prevalence, incidence and recovery probabilities. Dotted lines represent the expected values of naïve estimators when one sample is collected per unit. Solid lines represent the expected values of naïve estimators when three samples are collected per unit.

tives may arise due to a specific sample missing the pathogen (Level 3 above) or the failure of an assay to identify the pathogen in a positive sample (Level 4, Kirshtein et al. 2007). When false negative wetland assignments are possible, repeated sampling can provide information about detection probabilities to facilitate unbiased inferences about the spatial prevalence of $B d$ among wetlands. In the simple case in which false positives are not possible, a detection history of 011 would indicate an unambiguous $B d$ state assignment for the wetland: $B d$ was present in the wetland, but failed to be detected in the first of the three samples. A detection history of 000 is ambiguous because this observation can arise from two mutually exclusive wetland states: (1) the wetland was negative for the fungus; or (2) the wetland was positive for the fungus, but $B d$ was not detected in any of the samples. In essence, the repeat detection data for the unambiguous wetland state assignments can be used to inform probability statements about the true states of the ambiguous wetlands. This is achieved by modelling the detection histories as independent events arising from a multinomial distribution (MacKenzie et al. 2002). Any wetland $j$ with detection history $\mathbf{h}_{j}=011$ would have event probability

$\operatorname{Pr}\left(\mathbf{h}_{j}=011\right)=\psi\left(1-p_{1}\right) p_{2} p_{3}$,

where $\psi$ is the probability that a wetland within the park is $B d$ positive, and $p_{k}$ is the probability that $B d$ is detected in the kth sample (i.e., the sample contains the pathogen and it is detected by the assay), conditional on wetland $j$ being infected. The parameter $\psi$ can also be interpreted as the expected proportion (i.e., spatial prevalence) of $B d$ positive wetlands within the park. The ambiguous detection history $\mathbf{h}_{j}=000$ would have event probability 
$\operatorname{Pr}\left(\mathbf{h}_{j}=000\right)=\psi \prod_{k=1}^{3}\left(1-p_{k}\right)+(1-\psi)$,

where the additive terms account for the possibility that the wetland was $B d$ positive (but the fungus was not detected by sampling) or the wetland was truly $B d$ negative.

For any number of sample units and sampling occasions per unit, the likelihood function for this two-state system is simply the product of the event probabilities for each of the $s$ spatial units:

$L\left(\psi, \mathbf{p} \mid \mathbf{h}_{1}, \mathbf{h}_{2}, \ldots, \mathbf{h}_{s}\right) \propto \prod_{j=1}^{s} \operatorname{Pr}\left(\mathbf{h}_{j}\right)$.

The fundamental assumptions of this model are: (1) the infection state of each sample unit does not change during the period of sampling (e.g., an uninfected wetland does not become infected after the first sample or sampling occasion); (2) all units have the same probability of the pathogen being present; (3) the probability of a positive detection from a sample, given presence of the pathogen, is the same across all units; (4) detections are independent within and across units; and (5) there are no false positive detections. We will later describe how assumptions 2 and 3 may be relaxed, if appropriate covariates are available to model variation in prevalence and detection probabilities (see Incorporating more ecological realism). Similarly, assumption 5 may be relaxed, if information about the false positive detection process is available (see False positive state assignments). For this model (and all those to follow), the likelihood function and detection history data facilitate the estimation of parameters via maximum likelihood (e.g., MacKenzie et al. 2006) or Bayesian (e.g., Royle \& Dorazio 2008) analysis methods. All conditional probability statements in this paper result from integrating across the possible latent disease states for a given model (see Fig. 4). Alternatively, Monte Carlo integration methods can be used to the same effect (Royle \& Dorazio 2008).

The parameter $\psi$ thus corresponds to the prevalence of the pathogen among spatial units, an important parameter for inference about pattern in disease ecology. For inferences about pathogen dynamics, we will relax an assumption to examine changes in $B d$ spatial prevalence through time (Fig. 4). Suppose wetlands were sampled three times each year for two consecutive years, where $B d$ states were assumed static during within-year sampling but dynamic between years. The detection history $\mathbf{h}_{j}=101110$ would be unambiguous in wetland state assignment for both years, with event probability

$$
\begin{aligned}
\operatorname{Pr}\left(\mathbf{h}_{j}\right. & =101110) \\
& =\psi_{1} p_{1,1}\left(1-p_{1,2}\right) p_{1,3}\left(1-\phi_{1}^{[1,0]}\right) p_{2,1} p_{2,2}\left(1-p_{2,3}\right),
\end{aligned}
$$

where $\psi_{t}$ is $B d$ prevalence among wetlands within the park in year $t, p_{t, k}$ is the $B d$ detection probability for the kth sample of the $t$ th year, and $\phi_{t}^{[m, n]}$ is the probability of a wetland transitioning from state $m$ in year $t$ to state $n$ in year $t+1$ (see Table 2 ). The detection history $\mathbf{h}_{j}=000000$ would be ambiguous for both years, with probability

Event probability

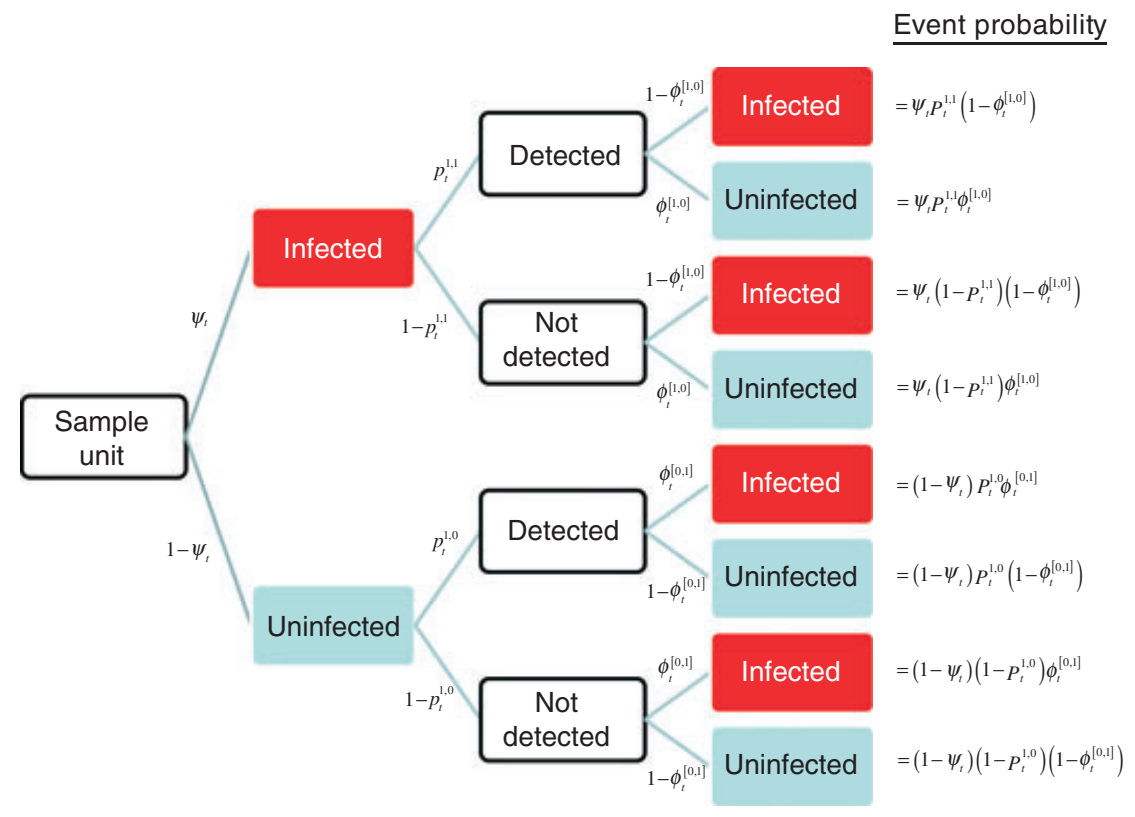

Figure 4 The possible event probability statements under a simple spatial disease dynamics model. A sample unit is infected at time $t$ with probability $\psi_{t}$, and given infection at time $t$, the unit is detected as infected with probability $p_{t}^{1,1}$. An uninfected unit may be (erroneously) detected as infected with probability $p_{t}^{1,0}$. By time $t+1$, an infected unit may have become uninfected with probability $\phi_{t}^{[1,0]}$, or an uninfected unit may have become infected with probability $\phi_{t}^{[0,1]}$. In application, it is typically assumed that $p_{t}^{1,0}=0$. See Table 2 for formal interpretation of parameter superscripts. 
Table 2 Definitions of parameters used in the hierarchical model of spatial disease effects, prevalence, and dynamics that incorporates state uncertainty. Additional spatial or sample subscripts may be added as required by the hierarchy

\begin{tabular}{ll}
\hline Parameter & Definition \\
\hline$\psi_{t}^{[m]}$ & $\begin{array}{c}\text { Probability of sample units within a larger } \\
\text { geographic area being in state } m \text { at time } t\end{array}$ \\
$\psi_{t}$ & Prevalence of infected sample units within \\
& a larger geographic area at time $t$ \\
$\phi_{t}^{[m, n]}$ & Probability of sample units within a larger \\
& geographic area transitioning from state \\
& $m$ at time $t$ to state $n$ at time $t+1$ \\
$\phi_{t}$ & Incidence of newly infected sample units within \\
& a larger geographic area from time $t$ to time \\
& $t+1$ \\
$p_{t, k}^{l, m}$ & Probability of detecting a sample unit in state \\
& $l$ in the kth sample collected at time $t$ when the \\
& true state is $m^{*}$
\end{tabular}

*In the absence of false positive errors, the state superscript is removed when there are only two states.

$$
\begin{aligned}
& \operatorname{Pr}\left(\mathbf{h}_{j}=000000\right)= \\
& \psi_{1} \prod_{k=1}^{3}\left(1-p_{1, k}\right)\left[\left(1-\phi_{1}^{[1,0]}\right) \prod_{k=1}^{3}\left(1-p_{2, k}\right)+\phi_{1}^{[1,0]}\right] \\
& \quad+\left(1-\psi_{1}\right)\left[\phi_{1}^{[0,1]} \prod_{k=1}^{3}\left(1-p_{2, k}\right)+\left(1-\phi_{1}^{[0,1]}\right)\right] .
\end{aligned}
$$

Spatial prevalence within the park after the first year can be obtained through the recursive equation $\psi_{t+1}=$ $\psi_{t}\left(1-\phi_{t}^{[1,0]}\right)+\left(1-\psi_{t}\right) \phi_{t}^{[0,1]}$ (MacKenzie et al. 2003). Under this parameterization, the spatial incidence $\left(\phi_{t}\right)$ among wetlands from time $t$ to time $t+1$ can be estimated by $\phi_{t}^{[0,1]}$.

\section{Simple host and pathogen dynamics}

These models can be extended beyond the simple two-state system that accommodates false negative detections for the pathogen. Suppose interest was not in whether specific wetlands were infected with $B d$, but instead in the effects, prevalence, and dynamics of $B d$ on an amphibian species potentially residing within these wetlands. Each wetland could then fall under one of at least three possible states: (1) unoccupied by the host species (State 0); (2) occupied by the host species with no infected individuals (State 1); and (3) occupied by the host species with infected individuals (State 2). Assuming no false positives, there is no uncertainty associated with a wetland's state assignment when an infected individual is detected. However, there remains uncertainty in the occupancy state of wetlands (due to nondetection of the amphibian species) and for the infection state assignment of occupied wetlands (due to non-detection of $B d$ ).

Consider the observed detection history $\mathbf{h}_{j}=012100$ for a wetland that was visited over 3 years, where individuals of an anuran species were captured (with replacement) and swabbed for $B d$ over two sampling periods within each year. In the first year, the anuran species was only detected on the second sampling period, and none of the sampled individuals tested positive for $B d$. The state of the wetland was partially observed because it is known that the wetland was occupied by the anuran species during both sampling occasions of the first year, but it is unclear whether the wetland actually had no infected individuals, or had infected individuals that sampling failed to detect. In the second year, the ' 2 ' indicates that infected individuals were detected during the first sampling occasion. It is therefore known that the wetland was occupied by infected individuals in the second year, with $B d$ failing to be detected on any of the individuals sampled during the second sampling occasion. No individuals were captured in the third year, and it is unclear whether the wetland was no longer occupied, occupied but uninfected, or occupied and infected. The 3 years of this detection history show a natural ordering of the degree of uncertainty about true wetland state. In year one, the wetland must be in either state 1 or 2 , whereas in year two the true wetland state is known to be 2. Year three admits the greatest uncertainty, with all three true states being possible. The probability of observing this detection history is

$$
\begin{aligned}
\operatorname{Pr}\left(\mathbf{h}_{j}=\right. & 012100) \\
= & \left(\psi_{1}^{[1]} p_{1,1}^{0,1} p_{1,2}^{1,1} \phi_{1}^{[1,2]}+\psi_{1}^{[2]} p_{1,1}^{0,2} p_{1,2}^{1,2} \phi_{1}^{[2,2]}\right) \\
& \times p_{2,1}^{2,2} p_{2,2}^{1,2}\left(\phi_{2}^{[2,0]}+\phi_{2}^{[2,1]} \prod_{k=1}^{2} p_{3, k}^{0,1}+\phi_{2}^{[2,2]} \prod_{k=1}^{2} p_{3, k}^{0,2}\right),
\end{aligned}
$$

where $\psi_{t}^{[m]}$ is the probability that a sample unit is in state $m$ in year $t, p_{t, k}^{l, m}$ is the probability of observing state $l$ on sampling occasion $k$ of year $t$ when the true state is $m$, and $\phi_{t}^{[m, n]}$ is the probability of a sample unit transitioning from state $m$ in year $t$ to state $n$ in year $t+1$ (MacKenzie et al. 2009). Note that in this example, we assume no false positives so that $p_{t, k}^{1,0}=p_{t, k}^{2,0}=p_{t, k}^{2,1}=0$.

Under this three-state system, $\psi_{t}^{[1]}+\psi_{t}^{[2]}$ is the probability of a wetland being occupied by the host species in year $t$, and the spatial prevalence of $B d$ among occupied wetlands is $\psi_{t}=\psi_{t}^{[2]} /\left(\psi_{t}^{[1]}+\psi_{t}^{[2]}\right), \quad$ where $\quad \psi_{t+1}^{[m]}=\psi_{t}^{[2]} \phi_{t}^{[2, m]}+$ $\psi_{t}^{[1]} \phi_{t}^{[1, m]}+\left(1-\psi_{t}^{[1]}-\psi_{t}^{[2]}\right) \phi_{t}^{[0, m]}$. The fraction of occupied sample units containing no infected individuals at time $t$ that contained infected individuals by time $t+1$ (i.e., spatial 
incidence $)$ is $\phi_{t}=\left[\psi_{t}^{[1]} \phi_{t}^{[1,2]}+\left(1-\psi_{t}^{[1]}-\psi_{t}^{[2]}\right) \phi_{t}^{[0,2]}\right] /$ $\left(1-\psi_{t}^{[2]}\right)$. As with all models herein, the likelihood for this dynamic three-state system accommodating non-detection is simply $L\left(\boldsymbol{\psi}, \boldsymbol{\phi}, \mathbf{p} \mid \mathbf{h}_{1}, \mathbf{h}_{2}, \ldots, \mathbf{h}_{s}\right) \propto \prod_{j=1}^{s} \operatorname{Pr}\left(\mathbf{h}_{j}\right)$. By directly incorporating state uncertainty, this approach to dynamical disease modelling not only allows changes in disease or pathogen prevalence across time and space to be reliably investigated, but also changes in host species occurrence as a function of disease or pathogen state.

\section{Hierarchical spatial patterns and dynamics}

We will now extend these models of disease state uncertainty to multiple spatial scales by incorporating additional levels of repeated sampling within Level 2 of the hierarchy (Fig. 2). Use of the same unified sampling framework through each level of the spatial hierarchy allows disease systems to be simultaneously examined across coarse where $\psi_{i}$ is the probability that park $i$ contains $B d$ (i.e., $B d$ prevalence among the parks within the broader geographic area), $\psi_{i, j}$ is the conditional probability that wetland $j$ contains $B d$ (i.e., $B d$ prevalence among the wetlands within park $i$, given the pathogen is present in park $i$ ), and $p_{k, i, j}$ is the probability that the kth sample from wetland $j$ in park $i$ is $B d$ positive and detected (Nichols et al. 2008; Kendall 2009).

Additional replication in time allows the spread of the pathogen to be examined at each of the spatial levels in the hierarchy. Suppose that after an additional year of sampling, the individual detection histories for the two sampled wetlands in park $i$ were $\mathbf{h}_{i, 1}=0000$ and $\mathbf{h}_{i, 2}=0100$. As $B d$ was not detected at either wetland in the second year, there is now uncertainty at both the park and wetland level. This can still be readily handled probabilistically at each level of the spatial hierarchy under the dynamical two-state model:

$$
\begin{aligned}
\operatorname{Pr}\left(\mathbf{h}_{i}=\begin{array}{ll}
00 & 0 \\
01 & 00
\end{array}\right)= & \psi_{1, i}\left(1-\phi_{1, i}^{[1,0]}\right)\left\{\begin{array}{l}
\left(\begin{array}{l}
\psi_{1, i, 1}\left(1-p_{1,1, i, 1}\right)\left(1-p_{1,2, i, 1}\right)\left[\left(1-\phi_{1, i, 1}^{[1,0]}\right)\left(1-p_{2,1, i, 1}\right)\left(1-p_{2,2, i, 1}\right)+\phi_{1, i, 1}^{[1,0]}\right] \\
+\left(1-\psi_{1, i, 1}\right)\left[\phi_{1, i, 1}^{[0,1]}\left(1-p_{2,1, i, 1}\right)\left(1-p_{2,2, i, 1}\right)+\left(1-\phi_{1, i, 1}^{[0,1]}\right)\right]
\end{array}\right) \\
\times \psi_{1, i, 2}\left(1-p_{1,1, i, 2}\right) p_{1,2, i, 2}\left[\left(1-\phi_{1, i, 2}^{[1,0]}\right)\left(1-p_{2,1, i, 2}\right)\left(1-p_{2,2, i, 2}\right)+\phi_{1, i, 2}^{[1,0]}\right]
\end{array}\right\} \\
+ & \psi_{1, i} \phi_{1, i}^{[1,0]}\left\{\begin{array}{l}
{\left[\begin{array}{l}
\left.\psi_{1, i, 1}\left(1-p_{1,1, i, 1}\right)\left(1-p_{1,2, i, 1}\right)+\left(1-\psi_{1, i, 1}\right)\right] \\
\times \psi_{1, i, 2}\left(1-p_{1,1, i, 2}\right) p_{1,2, i, 2}
\end{array}\right\}}
\end{array}\right.
\end{aligned}
$$

spatial units (e.g., for monitoring global trends) down to much finer units that may be of importance at the local population level. Perhaps instead of wetlands within a single park, interest lies in $B d$ within a system of parks covering a much broader geographic area (e.g., Muths et al. 2008). For simplicity, suppose two wetlands in each park were randomly sampled, and two water samples were collected from each wetland. If only one sample tested positive for $B d$, the individual detection histories for the two wetlands sampled in park $i$ could be $\mathbf{h}_{i, 1}=00$ and $\mathbf{h}_{i, 2}=01$. At the park level, the pathogen state is unambiguous because $B d$ was detected in at least one of the samples from at least one of the wetlands. However, there is ambiguity at the wetland level because the pathogen was not detected in either of the samples from the first wetland. Under the two-state hierarchical model, this joint detection history event for park $i$ would have probability

$$
\begin{aligned}
& \operatorname{Pr}\left(\mathbf{h}_{i}=\begin{array}{l}
00 \\
01
\end{array}\right)= \\
& \psi_{i}\left\{\begin{array}{l}
{\left[\psi_{i, 1}\left(1-p_{1, i, 1}\right)\left(1-p_{2, i, 1}\right)+\left(1-\psi_{i, 1}\right)\right]} \\
\times \psi_{i, 2}\left(1-p_{1, i, 2}\right) p_{2, i, 2}
\end{array}\right\},
\end{aligned}
$$

where $\psi_{t, i}$ is the probability that park $i$ contains $B d$ during year $t, \psi_{t, i, j}$ is the conditional probability that wetland $j$ contains $B d$ (given the pathogen is present in park $i$ ) during year $t, \phi_{t, i}^{[m, n]}$ is the probability of park $i$ transitioning from state $m$ in year $t$ to state $n$ in year $t+1, \phi_{t, i, j}^{[m, n]}$ is the probability of wetland $j$ transitioning from state $m$ in year $t$ to state $n$ in year $t+1$, and $p_{t, k, i, j}$ is the probability that the $k$ th sample from wetland $j$ of park $i$ is $B d$ positive and detected in year $t$. The first element on the right-hand side of the equation describes the wetland probability statements conditional on the park being $B d$ positive in both years. The second element describes the wetland probability statements conditional on the park becoming $B d$ negative, whereby all wetlands must be $B d$ negative in the second $\operatorname{year}\left(\phi_{1, i, j}^{[1,0]}=1-\phi_{1, i, j}^{[0,1]}=1\right)$. Prevalence at both scales can be obtained through the recursive equations $\psi_{t+1, i}=\psi_{t, i}\left(1-\phi_{t, i}^{[1,0]}\right)+\left(1-\psi_{t, i}\right) \phi_{t, i}^{[0,1]} \quad$ and $\psi_{t+1, i, j}=\left\{\psi_{t, i}\left(1-\phi_{t, i}^{[1,0]}\right)\left[\psi_{t, i, j}\left(1-\phi_{t, i, j}^{[1,0]}\right)+\left(1-\psi_{t, i, j}\right)\right.\right.$ $\left.\left.\phi_{t, i, j}^{[0,1]}\right]+\left(1-\psi_{t, i}\right) \phi_{t, i}^{[0,1]} \phi_{t, i, j}^{[0,1]}\right\} / \psi_{t+1, i}$. As before, 
$\phi_{t, i}=\phi_{t, i}^{[0,1]}$ is the spatial incidence among parks within the broader geographic area, or the probability of park $i$ transitioning from $B d$ negative in year $t$ to $B d$ positive in year $t+1$. Similarly, $\phi_{t, i, j}=\phi_{t, i, j}^{[0,1]}$ is the incidence among wetlands within park $i$, conditional on park $i$ being $B d$ positive in year $t+1$. We note that this model allows the possibility that a park is $B d$ positive, even if none of the sampled wetlands contains the pathogen (assuming not all wetlands within the park were sampled).

As eqn 2 implies, the hierarchical detection process and the likelihood that describes it can become quite complicated as the number of time periods, sample units, or sample occasions increases. Succinct matrix notation makes the model tractable:

$\operatorname{Pr}\left(\mathbf{h}_{i}\right)=\mathbf{y}\left[\prod_{t=1}^{T-1} \mathbf{D}_{t} \mathbf{R}_{t} \mathbf{W}_{t}\right] \mathbf{d}_{T}$

where $T$ is the number of sampling periods (e.g., years), $\mathbf{y}$ is an initial state vector, $\mathbf{D}_{t}$ is a diagonal matrix specifying the state-dependent probability of the observed detection history for all $s$ sample units at time $t, \mathbf{R}_{t}$ and $\mathbf{W}_{t}$ are the state transition probability matrices for the broader geographic area and the sample units, respectively, and $\mathbf{d}_{T}$ is a vector specifying the state-dependent probability of the observed detection history for all $s$ sample units at time $T$. Motivated readers are referred to Appendix S1 for complete details (see Supporting Information). in park $i$ during the first year yielded the detection histories $\mathbf{h}_{i, 1}=10$ and $\mathbf{h}_{i, 2}=21$, it is known that this park was occupied by infected individuals. Both wetlands were occupied by the species, but it is unclear whether the anuran population at the first wetland was infected or not. Observing this joint detection history has probability

$\operatorname{Pr}\left(\mathbf{h}_{i}=\begin{array}{l}10 \\ 21\end{array}\right)=\psi_{i}^{[2]}\left[\begin{array}{l}\left(\psi_{i, 1}^{[1]} p_{1, i, 1}^{1,1} p_{2, i, 1}^{0,1}+\psi_{i, 1}^{[2]} p_{1, i, 1}^{1,2} p_{2, i, 1}^{0,2}\right) \\ \times \psi_{i, 2}^{[2]} p_{1, i, 2}^{2,2} p_{2, i, 2}^{1,2}\end{array}\right]$,

where $\psi_{i}^{[m]}$ is the probability that park $i$ is in state $m, \psi_{i, j}^{[m]}$ is the probability that wetland $j$ is in state $m$, and $p_{k, i, j}^{l, m}$ is the probability of observing state $l$ for the $k$ th sample of wetland $j$ when the true wetland state is $m$. Under this model, $\psi_{i}=\psi_{i}^{[2]} /\left(\psi_{i}^{[1]}+\psi_{i}^{[2]}\right)$ is $B d$ prevalence among occupied parks within the broader geographic area, and $\psi_{i, j}=\psi_{i, j}^{[2]} /\left(\psi_{i, j}^{[1]}+\psi_{i, j}^{[2]}\right)$ is the conditional $B d$ prevalence among occupied wetlands within park $i$ (given park $i$ is infected). If in a second year of sampling the wetland detection histories $\mathbf{h}_{i, 1}=1000$ and $\mathbf{h}_{i, 2}=2111$ were observed, it is known that park $i$ remained occupied by the host (anuran) species, but it is unknown whether the park remained infected or became uninfected. At the lower level, it is also unknown whether either of the sampled wetlands was infected during the second year of sampling, or whether the first wetland remained occupied by the host species. The probability of observing this event under the hierarchical three-state dynamics model is

$$
\begin{aligned}
& \operatorname{Pr}\left(\mathbf{h}_{i}=\begin{array}{ll}
10 & 00 \\
21 & 11
\end{array}\right)=\psi_{1, i}^{[2]} \phi_{1, i}^{[2,1]}\left\{\begin{array}{l}
\left.\left[\begin{array}{l}
\psi_{1, i, 1}^{[1]} p_{1,1, i, 1}^{1,1} p_{1,2, i, 1}^{0,1}\left(\phi_{1, i, 1}^{[1,0]}+\phi_{1, i, 1}^{[1,1]} p_{2,1, i, 1}^{0,1} p_{2,2, i, 1}^{0,1}\right) \\
+\psi_{1, i, 1}^{[2]} p_{1,1, i, 1}^{1,2} p_{1,2, i, 1}^{0,2}\left(\phi_{1, i, 1}^{[2,0]}+\phi_{1, i, 1}^{[2,1]} p_{2,1, i, 1}^{0,1} p_{2,2, i, 1}^{0,1}\right)
\end{array}\right]\right\} \\
\times \psi_{1, i, 2}^{[2]} p_{1,1, i, 2}^{2,2} p_{1,2, i, 2}^{1,2} \phi_{1, i, 2}^{[2,1]} p_{2,1, i, 2}^{1,1} p_{2,2, i, 2}^{1,1}
\end{array}\right\} \\
& +\psi_{1, i}^{[2]} \phi_{1, i}^{[2,2]}\left\{\begin{array}{l}
{\left[\begin{array}{l}
\psi_{1, i, 1}^{[1]} p_{1,1, i, 1}^{1,1} p_{1,2, i, 1}^{0,1}\left(\phi_{1, i, 1}^{[1,0]}+\phi_{1, i, 1}^{[1,1]} p_{2,1, i, 1}^{0,1} p_{2,2, i, 1}^{0,1}+\phi_{1, i, 1}^{[1,2]} p_{2,1, i, 1}^{0,2} p_{2,2, i, 1}^{0,2}\right) \\
+\psi_{1, i, 1}^{[2]} p_{1,1, i, 1}^{1,2} p_{1,2, i, 1}^{0,2}\left(\phi_{1, i, 1}^{[2,0]}+\phi_{1, i, 1}^{[2,1]} p_{2,1, i, 1}^{0,1} p_{2,2, i, 1}^{0,1}+\phi_{1, i, 1}^{[2,2]} p_{2,1, i, 1}^{0,2} p_{2,2, i, 1}^{0,2}\right)
\end{array}\right]} \\
\times \psi_{1, i, 2}^{[2]} p_{1,1, i, 2}^{2,2} p_{1,2, i, 2}^{1,2}\left(\phi_{1, i, 2}^{[2,1]} p_{2,1, i, 2}^{1,1} p_{2,2, i, 2}^{1,1}+\phi_{1, i, 2}^{[2,2]} p_{2,1, i, 2}^{1,2} p_{2,2, i, 2}^{1,2}\right)
\end{array}\right\},
\end{aligned}
$$

\section{Hierarcbical multistate patterns and dynamics}

We now develop a hierarchical spatial model for the dynamical three-state system, where both sample units and larger geographic areas can be classified as unoccupied (State 0 ), occupied but uninfected (State 1), or occupied and infected (State 2) based on sampling of the host species. Returning to the $B d$ example, suppose sampling was conducted each year on two wetlands in each park, and anurans were captured and swabbed for $B d$ on two occasions within each year. If the two wetlands sampled where $\psi_{t, i}^{[m]}$ is the probability that park $i$ is in state $m$ during year $t, \psi_{t, i, j}^{[m]}$ is the probability that wetland $j$ of park $i$ is in state $m$ during year $t$, and $p_{t, k, i, j}^{l, m}$ is the probability of observing state $l$ in sample $k$ at wetland $j$ in year $t$ when the true state is $m$. The first element on the right-hand side of the equation describes the probability statements conditional on the park being occupied and infected in the first year, but becoming uninfected and remaining occupied by the host species in the second year. The second element describes the probability statements conditional on the park 
being occupied and infected during both years. As before, this model allows for a park to have been occupied by the host species and infected even if none of the sampled wetlands was. The model may also be defined in general terms using the matrix notation of eqn 3 by extending each component for an additional state (see Appendix S1 in Supporting Information).

The multistate hierarchical model is not limited to three states, and the sample units are not limited to unoccupied or occupied states for the host species. All sampling could occur at units occupied by the host, and the states could instead be defined only in terms of the pathogen (e.g., uninfected, less severe infection, more severe infection) to address questions about the spread and status of infections across host populations. One advantage of the former approach is that it allows the effects of disease or infection states to be assessed through the patterns and dynamics of the host species. For example, if the host populations of infected units tend to go locally extinct at a greater rate than those of uninfected units $\left(\phi^{[2,0]}>\phi^{[1,0]}\right)$, then this provides evidence for the negative impacts of the pathogen. This approach would be particularly useful for large scale $B d$ monitoring programs, because it is not yet understood why some infected regions are exhibiting local declines while other infected regions are not, as demonstrated in boreal toad (Bufo boreas) populations in the southern and northern Rocky Mountains (Scherer et al. 2005; Muths et al. 2008).

Despite our focus thus far on uncertainty within spatial levels, the hierarchy need not be limited to levels in space. For example, additional replication within Level 3 of the hierarchy at the individual sample level (Fig. 1b, steps 3-5) allows the probability that a sample contains a pathogen (given the unit is infected) to be distinguished from the probability that a pathogen is detected by the assay technique(s), given the sample contained the pathogen. This information could be particularly useful for examining infection prevalence within local populations or for designing optimal sampling and laboratory protocols.

\section{Incorporating more ecological realism}

All of these models may be further tailored to address specific ecological hypotheses about the factors driving pathogen prevalence and dynamics. For example, if $B d$ prevalence is believed to be associated with temperature, one could parameterize prevalence as a function of average seasonal temperature using a logistic regression (e.g., Martin et al. 2005) such that:

$\operatorname{logit}\left(\psi_{i}\right)=\beta_{0}+\beta_{T} x_{i}$,

where $\beta_{0}$ is an intercept term, $\beta_{T}$ is a term for the temperature effect, and $x_{i}$ is the average temperature for park $i$. This approach could also be used to model transition probabilities between states (e.g., incidence) as a function of one or more covariates. Autologistic models (Besag 1972) may be incorporated within this context, in that the infection state of a spatial unit may be correlated with that of its neighbours. For example, one could model incidence in the two-state model as

$\operatorname{logit}\left(\phi_{i, j}^{[0,1]}\right)=\beta_{0}+\lambda_{1}\left(z_{i-1, j}+z_{i+1, j}\right)+\lambda_{2}\left(z_{i, j-1}+z_{i, j+1}\right)$,

where the probability that an uninfected unit in the ith row and $j$ th column of a spatial lattice becomes infected is related to prevalence $\left(z, j=\psi_{i, j}\right)$ or infection state $\left(z_{i, j}=0,1\right)$ at neighbouring units, and $\lambda_{k}$ is a term for the spatial dependence in direction $k$. Because $q_{i, j}$ is not a standard covariate, this parameter must be estimated to account for imperfect detection. Simulation-based Bayesian approaches utilizing Markov chain Monte Carlo (MCMC, e.g., Royle \& Dorazio 2008) appear to be especially well-suited for the simultaneous estimation of parameters for the set of locations in the sampled region. A less mechanistic approach could utilize spatially correlated random effects (Magoun et al. 2007).

One could also model spatial dependence as a function of the distance to occupied or infected neighbours. For example, prevalence in the two-state model could be parameterized as

$\operatorname{logit}\left(\psi_{i}\right)=\beta_{0}+\beta_{D}\left(\frac{\sum_{j=1}^{k_{i}} w_{i j} z_{j}}{\sum_{j=1}^{k_{i}} w_{i j}}\right)$,

where $\beta_{D}$ is a spatial dependence term for unit $i, z_{j}$ is the prevalence $\left(z_{j}=\psi_{j}\right)$ or infection state $\left(z_{j}=0,1\right)$ for unit $j$ within a set of $k_{i}$ neighbouring units, $w_{i j}=1 / h_{i j}$ is the weight given to unit $j$, and $h_{i j}$ is the Euclidian distance between units $i$ and $j$ (Augustin et al. 1996). As above, the $z_{j}$ covariate must be estimated under imperfect detection, and simultaneous estimation of $z_{j}$ and $\psi_{i}$ can be accomplished using MCMC. As a metric of disease spread, these methods would also be useful for modelling incidence as a function of distance to infected neighbours.

Many other sources of information can be readily incorporated into the repeated sampling framework. For example, one could collect detection data using multiple methods (e.g., water and anuran swab samples) to examine prevalence at each of the corresponding scales (e.g., wetland and individual level) sensu Nichols et al. (2008). Within a disease context, models of species cooccurrence patterns and dynamics have been underutilized due to a lack of suitable data sets (Gotelli \& Rohde 2002). However, the proposed hierarchy can be extended to recently developed co-occurrence models when species are detected imperfectly (MacKenzie et al. 2006). As the 
most important predictors may differ by spatial scale, one could also investigate different explanatory variables at each level of the hierarchy. These could include variables related to heterogeneity in detection probability parameters, a potential source of observability bias if not accounted for in these models (Royle 2006; Jennelle et al. 2007). This flexibility allows many interesting hypotheses to be explored within the same probabilistic framework, with model selection and multimodel inference procedures available (e.g., Hoeting et al. 1999; Burnham \& Anderson 2002) as weights of evidence for competing hypotheses about the specific system of interest (see Plowright et al. 2008 for applications in disease ecology).

\section{False positive state assignments}

We have thus far proposed a general strategy for incorporating uncertainty into hierarchical spatial models of disease. The specification of these models is straightforward and only requires some form of repeated sampling at each level of the hierarchy. However, one problem of this approach is the assumption of no misclassification due to false positive errors when assigning disease or occupancy states. This assumption provides one unambiguous state (i.e., a gold standard) for which truth is 'known', leading to the ability to estimate other model parameters in the face of substantial uncertainty due to non-detection. False positive errors have received considerably less attention in other fields of ecology (but see Royle \& Link 2006), and are arguably the most difficult to account for because they remove the unambiguous observations on which estimation is anchored. Despite the difficulty, disease studies present a special opportunity to address false positives because there is the potential to minimize or quantify the frequency of these errors through laboratory procedures.

The potential for false positive errors can be greatly reduced through sound laboratory practices. Clearly, these would include proper handling of samples and equipment to prevent contamination, but also the selection of fresh samples in the field. Optimizing tests for sensitivity and specificity based on known negative and positive samples is a standard procedure, and perhaps the best strategy for reducing false positive errors is to calibrate for lower sensitivity (Chua et al. 2007) and higher specificity (VanDalen et al. 2008). This approach would result in more false negative detections and create new problems for inference methods that do not incorporate nondetection, but these issues are readily handled by models such as those we have proposed. Indeed, the meticulous design and implementation of laboratory protocols can greatly reduce the propensity for false positives, but it is very unlikely that protocols alone will completely eliminate them. It therefore seems warranted to incorporate false positive errors within the hierarchical modelling framework, and this theme constitutes a promising area for future research.

Instead of relying on more expensive laboratory diagnostics, disease or infection states are sometimes assigned based on visual inspection in the field (Jennelle et al. 2007; Conn \& Cooch 2009). Depending on how these states are defined, there is arguably greater potential for misclassification when using visual inspection instead of laboratory diagnostics. One approach to minimizing the potential for false positive errors is to define the observed infected states conservatively and rely on models accounting for non-detection to correct for the these errors. Alternatively, a subset of randomly selected individuals could be diagnosed using both field and laboratory diagnostics, where the laboratory state assignment is considered a reference from which to assess the misclassification errors of the field diagnostic method (e.g., Hui \& Walter 1980). This information could then be directly incorporated within Level 4 of the hierarchy (Fig. 2).

Another potential avenue uses ancillary information about false positive error rates based on expert prior information (e.g., Branscum et al. 2004) or additional tests in the laboratory. Suppose a calibration protocol is established to optimize sensitivity and specificity based on samples of known infection state. If there were concerns about contamination or if the procedure deemed 'optimal' still permitted the occasional false positive, this probability could be estimated based on repeated tests of known negative samples. The outcomes of $n$ independent trials could be assumed binomially distributed, with false positive assignment probability $p^{1,0}$ (Fig. 4). This auxiliary information could then be incorporated into the models developed above by constructing a joint likelihood for the detection histories incorporating false positive errors. Under the twostate hierarchical model (eqn 1), the individual sample unit detection histories $\mathbf{h}_{i, 1}=00$ and $\mathbf{h}_{i, 2}=01$ would have joint event probability

$$
\begin{aligned}
& \operatorname{Pr}\left(\mathbf{h}_{i}=\begin{array}{c}
00 \\
01
\end{array}\right) \\
& =\psi_{i}\left\{\begin{array}{l}
{\left[\psi_{i, 1}\left(1-p_{1, i, 1}^{1,1}\right)\left(1-p_{2, i, 1}^{1,1}\right)+\left(1-\psi_{i, 1}\right)\left(1-p^{1,0}\right)^{2}\right]} \\
\times\left[\psi_{i, 2}\left(1-p_{1, i, 2}^{1,1}\right) p_{2, i, 2}^{1,1}+\left(1-\psi_{i, 2}\right)\left(1-p^{1,0}\right)\left(p^{1,0}\right)\right]
\end{array}\right\} \\
& +\left(1-\psi_{i}\right)\left(1-p^{1,0}\right)^{3}\left(p^{1,0}\right),
\end{aligned}
$$

where $p_{k, i, j}^{l, m}$ is the probability of observing state $l$ in sample $k$ of unit $j$ within larger geographic area $i$ when the true unit state is $m$. The joint likelihood for this model is 


$$
\begin{aligned}
& L\left(\boldsymbol{\psi}, \mathbf{p}^{1,1}, p^{1,0} \mid \mathbf{h}_{1}, \mathbf{h}_{2}, \ldots, \mathbf{h}_{s}, y, n\right) \\
& \propto\left(p^{1,0}\right)^{y}\left(1-p^{1,0}\right)^{n-y} \prod_{i=1}^{S} \operatorname{Pr}\left(\mathbf{h}_{i} \mid p^{1,0}\right),
\end{aligned}
$$

where $y$ is the number of false positive events during the laboratory trials. Equation 1 then arises as a special case of this model under the constraint $p^{1,0}=0$.

\section{CONCLUSION}

Methods originally developed in other areas of ecology are clearly extendable to disease surveillance, monitoring, and modelling in ways that have yet to be widely appreciated by disease ecologists. We believe that hierarchical occupancy models provide a natural framework for inference about disease prevalence and dynamics across time and space, in the same way that multistate capture-recapture models provide a natural way to investigate rates of mortality and disease transmission among individuals within a local population. Through repeated sampling, this general framework allows a diverse range of questions about disease effects, prevalence, and dynamics to be addressed while rigorously accounting for uncertainty induced by observation error. We do not claim that adoption of our suggestions will provide a complete remedy for the 'paucity of understanding of wildlife disease epidemiology', as a general sampling and modelling framework is one of several components for which improvements are needed (Gulland 1995). However, we believe that use of these approaches will represent a large step in the right direction. Just as patients are encouraged to seek a second medical opinion before beginning treatment, we encourage disease ecologists to invest in repeated sampling before drawing inferences.

Perhaps our most important messages are that disease ecologists are seldom (if ever) able to perfectly detect disease, and that properly accounting for this inability is critical to reliable inference. Non-detection and misclassification errors are not statistical fine points, but rather can produce misleading inferences if not properly incorporated into inference methods (e.g., Martin et al. 2005; Royle \& Link 2006). This recognition of the importance of detection issues took many years to be adopted in animal population and community ecology; we do not expect rapid adoption of this methodology in disease ecology. Yet we do hope for similar changes to occur in disease ecology that will lead to more reliable inferences about pathogens and disease in natural systems.

We anticipate the arguments that the proposed modelling framework requires additional data, and the nature of the repeated sampling design will likely incur additional costs in the field or laboratory. Indeed, this may pose a significant hurdle because available resources are often inadequate for implementing epidemiological studies at the scales these models allow. However, we suspect there are many cases where repeated sampling is conducted as part of field or laboratory protocols, but not utilized for the estimation of detection probabilities (e.g., Begon et al. 1998; Atkinson et al. 2005; Salkeld \& Schwarzkopf 2005; McLean et al. 2007). For existing surveillance and monitoring programs where study costs are fixed, the need for replicate sampling at sample units will sometimes result in fewer units being sampled. This should not be viewed as undesirable. Under imperfect detection, MacKenzie \& Royle (2005) demonstrated that without sufficient replicate surveys, sampling more units can actually result in less precise estimates of prevalence than sampling fewer units with a more appropriate level of replication. Similar results will likely hold for the more complicated hierarchical models introduced here.

We emphasize that failing to obtain replicate samples from the unit of interest leads to inferences about a random variable (disease detection) that confound both: (1) true disease presence; and (2) sampling and detection processes. We believe that inference about true disease pattern and process based on a smaller number of units will virtually always be preferable to inference about a confounded variable based on a larger number of units. Moreover, provision of a clear framework for sampling and inference leads naturally to the development of efficient sampling designs that are optimal with respect to study objectives (e.g., MacKenzie \& Royle 2005). For example, the ability to combine data from visual assessments of infection with high misclassification error and more expensive laboratory assays permitting unambiguous disease classification should permit useful recommendations about the optimal mix of effort devoted to these low expense/high error and high expense/low error survey methods. In many cases, welldesigned approaches may ultimately cost less and provide more understanding than a composite of multiple uncoordinated, opportunistic surveillance protocols.

We also anticipate reluctance of wildlife disease ecologists to embrace models and inference methods that appear to be more complicated than those previously used. We do not deny that incorporation of parameters reflecting detection and misclassification lead to additional complexity. However, we note that some of the simpler models presented in this paper can be implemented using available software, such as programs PRESENCE (Hines 2006) and MARK (White \& Burnham 1999). Both PRESENCE and MARK rely primarily on a maximum likelihood framework for inference, but Bayesian analyses can be readily implemented using MCMC approaches (e.g., Royle \& Dorazio 2008; MacKenzie et al. 2009). As ecologists show interest in these types of models, we anticipate the rapid development of software to implement additional specific models that are useful in epidemiology. 
Use of hierarchical models of the type presented here will permit inferences relevant to a number of important questions in spatial epidemiology and disease ecology. Single-season models (i.e., those with no temporal component) permit inferences about spatial patterns of pathogens and disease, as well as about ecological and environmental covariates associated with spatial variation. The multiseason models permit inferences about pathogen and disease dynamics over both time and space. Factors affecting disease outbreaks and remissions are the subject of many of the more interesting ecological hypotheses, so we anticipate much interest in covariate modelling of these pathogen- and host-level vital rates. Multistate, multiseason models permit simultaneous inference about disease and its effects on the occurrence dynamics of host populations, a topic important to conservation biology and evolutionary ecology.

We also believe that hierarchical disease models hold great promise for wildlife disease management. Management decisions may involve diverse actions, ranging from vaccination to depopulation of infected individuals. Informed decision making will require models projecting the effects of different management actions on the vital rates of the disease and host populations (e.g., Williams et al. 2002). The inference methods presented here would be useful in the initial development of such models. They would be especially useful for monitoring consequences of actions by providing estimates of disease-dynamic parameters to discriminate among competing models of management effects.

\section{ACKNOWLEDGEMENTS}

This research was funded by the U.S. Geological Survey Amphibian Research and Monitoring Initiative. We thank Ian Goudie for helpful discussions on technical aspects of this work.

\section{REFERENCES}

Anderson, D.R. (2001). The need to get the basics right in wildlife field studies. Wildlife Soc. B, 29, 1294-1297.

Atkinson, C.T., Lease, J.K., Dusek, R.J. \& Samuel, M.D. (2005). Prevalence of pox-like lesions and malaria in forest bird communities on leeward Mauna Loa volcano, Hawaii. Condor, 107, 537-546.

Augustin, N.H., Mugglestone, M.A. \& Buckland, S.T. (1996). An autologistic model for the spatial distribution of wildlife. J. Appl. Ecol., 33, 339-347.

Barlow, N.D. (1995). Critical evaluation of wildlife disease models. In: Ecology of Infectious Diseases in Natural Populations (eds Grenfell, B.T. \& Dobson, A.P.). Cambridge University Press, Cambridge, pp. 230-259.

Begon, M., Feore, S.M., Bown, K., Chantrey, J., Jones, T. \& Bennett, M. (1998). Population and transmission dynamics of cowpox in bank voles: testing fundamental assumptions. Ecol. Lett., 1, 82-86.

Besag, J. (1972). Nearest-neighbour systems and the auto-logistic model for binary data. J. Roy. Stat. Soc. B, 34, 75-83.

Branscum, A.J., Gardner, I.A. \& Johnson, W.O. (2004). Bayesian modeling of animal- and herd-level prevalences. Prev. Vet. Med., 66, 101-112.

Brown, J.D., Stallknecht, D.E., Beck, J.R., Suarez, D.L. \& Swayne, D.E. (2006). Susceptibility of North American ducks and gulls to H5N1 highly pathogenic avian influenza viruses. Emerg. Infect. Dis., 12, 1663-1670.

Burnham, K.P. \& Anderson, D.R. (2002). Model Selection and Multimodel Inference: An Information-Theoretic Approach, 2nd edn. Springer-Verlag, New York.

Carey, C., Bruzgul, J.E., Livo, L.J., Walling, M.L., Kuehl, K.A., Dixon, B.F. et al. (2006). Experimental exposures of boreal toads (Bufo boreas) to a pathogenic chytrid fungus (Batrachochytrium dendrobatidis). EcoHealth, 3, 5-21.

Cattoli, G. \& Capua, I. (2007). Diagnosing avian influenza in the framework of wildlife surveillance efforts and environmental samples. J. Wildlife Dis., 43, S35-S39.

Chua, T.-H., Ellis, T.M., Wong, C.W., Guan, Y., Ge, S.X., Peng, G. et al. (2007). Performance evaluation of five detection tests for avian influenza antigen with various avian samples. Avian Dis., 51, 96-105.

Clark, L. \& Hall, J. (2006). Avian influenza in wild birds: status as reservoirs, and risks to humans and agriculture. Ornithol. Monogr., 60, 3-29.

Conn, P.B. \& Cooch, E.G. (2009). Multi-state capture-recapture analysis under imperfect state observation: an application to disease models. J. Appl. Ecol., 46, 486-492.

Conner, M.M., McCarty, C.W. \& Miller, M.W. (2000). Detection of bias in harvest-based estimates of chronic wasting disease prevalence in mule deer. J. Wildlife Dis., 36, 691-699.

Dobson, A.P. \& Foufopoulos, J. (2001). Emerging infectious pathogens of wildlife. Philos. T. Roy. Soc. B, 356, 1001-1012.

Faustino, C.R., Jennelle, C.S., Connolly, V., Davis, A.K., Swarthout, E.C., Dhondt, A.A. et al. (2004). Mycoplasma gallisepticum infection dynamics in a house finch population: seasonal variation in survival, encounter and transmission rate. J. Anim. Ecol., 73, 651-669.

Gotelli, N.J. \& Rohde, K. (2002). Co-occurrence of ectoparasites of marine fishes: a null model analysis. Ecol. Lett., 5, 86-94.

Gulland, F.M.D. (1995). The impact of infectious diseases on wild animal populations - a review. In: Ecology of Infectious Diseases in Natural Populations (eds Grenfell, B.T. \& Dobson, A.P.). Cambridge University Press, Cambridge, pp. 20-51.

Hartup, B.K., Bickal, J.M., Dhondt, A.A., Ley, D.H. \& Kollias, G.V. (2001). Dynamics of conjunctivitis and Mycoplasma gallisepticum infections in house finches. Auk, 118, 327-333.

Hess, G.R., Randolph, S.E., Arneberg, P., Chemini, C., Furlanello, C., Harwood, J. et al. (2002). Spatial aspects of disease dynamics. In: The Ecology of Wildlife Diseases (eds Hudson, P.J., Rizzoli, A., Grenfell, B.T., Heesterbeek, H. \& Dobson, A.P.). Oxford University Press, Oxford, pp. 102-118.

Hines, J.E. (2006). PRESENCE2 - software to estimate patch occupancy and related parameters. USGS-PWRC. [WWW document]. Available at: http://www.mbr-pwrc.usgs.gov/software/ presence.html. 
Hoeting, J.A., Madigan, D.M., Raftery, A.E. \& Volinsky, C.T. (1999). Bayesian model averaging: a tutorial. Stat. Sci., 14, 382401.

Hudson, P.J., Dobson, A.P. \& Newborn, D. (1998). Prevention of population cycles by parasite removal. Science, 282, 2256-2258.

Hudson, P.J., Rizzoli, A., Grenfell, B.T., Heesterbeek, H. \& Dobson, A.P. (2002). The Ecology of Wildlife Diseases. Oxford University Press, Oxford.

Hui, S.L. \& Walter, S.D. (1980). Estimating the error rates of diagnostic tests. Biometrics, 36, 167-171.

Hyatt, A.D., Boyle, D.G., Olsen, V., Boyle, D.B., Berger, L., Obendorf, D. et al. (2007). Diagnostic assays and sampling protocols for the detection of Batrachochytrium dendrobatidis. Dis. Aquat. Organ., 73, 175-192.

Jennelle, C.S., Cooch, E.G., Conroy, M.J. \& Senar, J.C. (2007). State-specific detection probabilities and disease prevalence. Ecol. Appl., 17, 154-167.

Johnson, M.L. \& Speare, R. (2003). Survival of Batrachochytrium dendrobatidis in water: quarantine and disease control implications. Emerg. Infect. Dis., 9, 922-925.

Joly, D.O. \& Messier, F. (2004). Factors affecting apparent prevalence of tuberculosis and brucellosis in wood bison. J. Anim. Ecol., 73, 623-631.

Jones, K.E., Patel, N.G., Levy, M.A., Storeygard, A., Balk, D., Gittleman, J.L. et al. (2008). Global trends in emerging infectious diseases. Nature, 451, 990-994.

Jourdain, E., Gauthier-Clerc, M., Bicout, D.J. \& Sabatier, P. (2007). Bird migration routes and risk for pathogen dispersion into Western Mediterranean wetlands. Emerg. Infect. Dis., 13, 365-372.

Keeling, M.J. \& Rohani, P. (2007). Modeling Infectious Diseases in Humans and Animals. Princeton University Press, Princeton.

Kendall, W.L. (2009). One size does not fit all: adapting markrecapture and occupancy models for state uncertainty. In: Modeling Demographic Processes in Marked Populations (eds Thomson, D.L., Cooch, E.G. \& Conroy, M.J.). Springer, New York, pp. 765-780.

Kendall, W.L. \& White, G.C. (2009). A cautionary note on substituting spatial subunits for repeated temporal sampling in studies of site occupancy. J. Appl. Ecol., 46, 1182-1188.

Kirshtein, J.D., Anderson, C.W., Wood, J.S., Longcore, J.E. \& Voytek, M.A. (2007). Quantitative PCR detection of Batrachochytrium dendrobatidis DNA from sediments and water. Dis. Aquat Organ., 77, 11-15.

Liu, J., Xiao, H., Lei, F., Zhu, Q., Qin, K., Zhang, X.-W. et al. (2005). Highly pathogenic H5N1 influenza virus infection in migratory birds. Science, 309, 1206.

Lukacs, P.M. \& Burnham, K.P. (2005). Estimating population size from DNA-based closed capture-recapture data incorporating genotyping error. J. Wildlife Manage., 69, 396-403.

MacKenzie, D.I. \& Royle, J.A. (2005). Designing occupancy studies: general advice and allocating survey effort. J. Appl. Ecol., 42, 1105-1114.

MacKenzie, D.I., Nichols, J.D., Lachman, G.B., Droege, S., Royle, J.A. \& Langtimm, C.A. (2002). Estimating site occupancy rates when detection probabilities are less than one. Ecology, 83, 22482255.

MacKenzie, D.I., Nichols, J.D., Hines, J.E., Knutson, M.G. \& Franklin, A.B. (2003). Estimating site occupancy, colonization, and local extinction when a species is detected imperfectly. Ecology, 84, 2200-2207.
MacKenzie, D.I., Nichols, J.D., Royle, J.A., Pollock, K.H., Bailey, L.L. \& Hines, J.E. (2006). Occupancy Estimation and Modeling: Inferring Patterns and Dynamics of Species Occurrence. Academic Press, New York.

MacKenzie, D.I., Nichols, J.D., Seamans, M.E. \& Gutiérrez, R.J. (2009). Modeling species occurrence dynamics with multiple states and imperfect detection. Ecology, 90, 823-835.

Magoun, A.J., Ray, J.C., Johnson, D.S., Valkenburg, P., Dawson, F.N. \& Bowman, J. (2007). Modeling wolverine occurrence using aerial surveys of tracks in snow. J. Wildlife Manage., 71, 2221-2229.

Martin, T.G., Wintle, B.A., Rhodes, J.R., Kuhnert, P.M., Field, S.A., Low-Choy, S.J. et al. (2005). Zero tolerance ecology: improving ecological inference by modelling the source of zero observations. Ecol. Lett., 8, 1235-1246.

McLean, R.G., Hall, J.S., Franklin, A.B., Sullivan, H., VanDalen, K., Shriner, S. et al. (2007). Avian influenza in wild birds: environmental sampling for the rapid detection of avian influenza viruses. Proc. Wildl. Damage Manage. Conf., 12, 87-93.

Mellor, A.A. \& Rockwell, R.F. (2006). Habitat shifts and parasite loads of lesser snow geese (Chen caerulescens caerulescens). ÉcoScience, 13, 497-502.

Muths, E., Pilliod, D.S. \& Livo, L.J. (2008). Distribution and environmental limitations of an amphibian pathogen in the Rocky Mountains, USA. Biol. Conserv., 141, 1484-1492.

Nichols, J.D., Bailey, L.L., O’Connell, A.F., Talancy, N.W., Grant, E.H.C., Gilbert, A.T. et al. (2008). Multi-scale occupancy estimation and modeling using multiple detection methods. J. Appl. Ecol., 45, 1321-1329.

Norman, S.A. (2008). Spatial epidemiology and GIS in marine mammal conservation medicine and disease research. EcoHealth, 5, 257-267.

Nusser, S.M., Clark, W.R., Otis, D.L. \& Huang, L. (2008). Sampling considerations for disease surveillance in wildlife populations. J. Wildlife Manage., 72, 52-60.

Olsen, B., Munster, V.J., Wallensten, A., Waldenström, J., Osterhaus, A.D.M.E. \& Fouchier, R.A.M. (2006). Global patterns of influenza A virus in wild birds. Science, 312, 384-388.

Osnas, E.E., Heisey, D.M., Rolley, R.E. \& Samuel, M.D. (2009). Spatial and temporal patterns of chronic wasting disease: finescale mapping of a wildlife epidemic in Wisconsin. Ecol. Appl., 19, 1311-1322.

Ostfeld, R.S. \& Holt, R.D. (2004). Are predators good for your health? Evaluating evidence for top-down regulation of zoonotic disease reservoirs Front. Ecol. Environ., 2, 13-20.

Packer, C., Holt, R.D., Hudson, P.J., Lafferty, K.D. \& Dobson, A.P. (2003). Keeping the herds healthy and alert: implications of predator control for infectious disease. Ecol. Lett., 6, 797-802.

Plowright, W. (1988). Research on wildlife diseases: is a reappraisal necessary? Rev. Sci. Tech., 7, 783-795.

Plowright, R.K., Sokolow, S.H., Gorman, M.E., Daszak, P. \& Foley, J.E. (2008). Causal inference in disease ecology: investigating ecological drivers of disease emergence. Front. Ecol. Environ., 6, 420-429.

Pounds, J.A., Bustamante, M.R., Coloma, L.A., Consuegra, J.A., Fogden, M.P.L., Foster, P.N. et al. (2006). Widespread amphibian extinctions from epidemic disease driven by global warming. Nature, 439, 161-167.

Royle, J.A. (2006). Site occupancy models with heterogeneous detection probabilities. Biometrics, 62, 97-102. 
Royle, J.A. \& Dorazio, R.M. (2008). Hierarchical Modeling and Inference in Ecology. Academic Press, San Diego.

Royle, J.A. \& Link, W.A. (2005). A general class of multinomial mixture models for anuran calling survey data. Ecology, 86, 25052512.

Royle, J.A. \& Link, W.A. (2006). Generalized site occupancy models allowing for false positive and false negative errors. Ecology, 87, 835-841.

Salkeld, D.J. \& Schwarzkopf, L. (2005). Epizootiology of blood parasites in an Australian lizard: a mark-recapture study of a natural population. Int. J. Parasitol., 35, 11-18.

Salman, M.D. (2003). Surveillance and monitoring systems for animal health programs and disease surveys. In: Animal Disease Surveillance and Survey Systems: Methods and Applications (ed Salman, M.D.). Iowa State University Press, Ames, pp. 3-14.

Schall, J.J., Pearson, A.R. \& Perkins, S.L. (2000). Prevalence of malaria parasites (Plasmodium floridense and Plasmodium azurophilum) infecting a Puerto Rican lizard (Anolis gundlachi): a nine-year study. J. Parasitol., 86, 511-515.

Scherer, R.D., Muths, E., Noon, B.R. \& Corn, P.S. (2005). An evaluation of weather and disease as causes of decline in two populations of boreal toads. Ecol. Appl., 15, 2150-2160.

Smith, K.F., Sax, D.E. \& Lafferty, K.D. (2006). Evidence for the role of infectious diseases in species extinction and endangerment. Conserv. Biol., 20, 1349-1357.

Smith, K.G., Lips, K.R. \& Chase, J.M. (2009). Selecting for extinction: nonrandom disease-associated extinction homogenizes amphibian biotas. Ecol. Lett., 12, 1-10.

Taylor, L.H., Latham, S.M. \& Woolhouse, M.E.J. (2001). Risk factors for human disease emergence. Philos. T. Roy. Soc. B, 356, 983-989.

U. S. Interagency Strategic Plan. (2006). An early detection system for highly pathogenic H5N1 avian influenza in wild migratory birds. [WWW document]. Available at: http://www.usda.gov/ documents/wildbirdstrategicplanpdf.pdf.

VanDalen, K.K., Anderson, T.D., Killian, M.L., Pedersen, J.C., Franklin, A.B. \& Piaggio, A.J. (2008). Increased detection of influenza A H16 in the United States. Arch. Virol., 153, 19811983.

Wake, D.B. \& Vredenburg, V.T. (2008). Are we in the midst of the sixth mass extinction? A view from the world of amphibians. Proc. Natl. Acad. Sci. USA, 105, 11466-11473.

Wallenstein, A., Munster, V.J., Latorre-Margalef, N., Brytting, M., Elmberg, J., Fouchier, R.A.M. et al. (2007). Surveillance of influenza A virus in migratory waterfowl in northern Europe. Emerg. Infect. Dis., 13, 404-411.
Ward, M.R., Stallknecht, D.E., Willis, J., Conroy, M.J. \& Davidson, W.R. (2006). Wild bird mortality and west Nile virus surveillance: biases associated with detection, reporting, and carcass persistence. J. Wildlife Dis., 42, 92-106.

Webster, R.G., Bean, W.J., Gorman, O.T., Chambers, T.M. \& Kawaoka, Y. (1992). Evolution and ecology of influenza A viruses. Microbiol. Rev., 56, 152-179.

Webster, R.G., Peiris, M., Chen, H. \& Guan, Y. (2006). H5N1 outbreaks and enzootic influenza. Emerg. Infect. Dis., 12, 3-8.

White, G.C. \& Burnham, K.P. (1999). Program MARK: survival estimation from populations of marked individuals. Bird Study, 46, 120-139.

Williams, B.K., Nichols, J.D. \& Conroy, M.J. (2002). Analysis and Management of Animal Populations. Academic Press, San Diego.

Wobeser, G.A. (2006). Essentials of Disease in Wild Animals. Blackwell Publishing, Ames.

Yasué, M., Feare, C.J., Bennun, L. \& Fieldler, W. (2006). The epidemiology of H5N1 avian influenza in wild birds: why we need better ecological data. Bioscience, 56, 923-929.

Yoshizaki, J., Pollock, K.H., Brownie, C. \& Webster, R.A. (2009). Modeling misidentification errors in capture-recapture studies using photographic identification of evolving marks. Ecology, 90, 3-9.

\section{SUPPORTING INFORMATION}

Additional Supporting Information may be found in the online version of this article:

Appendix S1 Matrix representation of the hierarchical model.

As a service to our authors and readers, this journal provides supporting information supplied by the authors. Such materials are peer-reviewed and may be re-organized for online delivery, but are not copy-edited or typeset. Technical support issues arising from supporting information (other than missing files) should be addressed to the authors.

Editor, Frederick Adler

Manuscript received 3 November 2009

First decision made 9 December 2009

Manuscript accepted 24 February 2010 